Preference is given to letters commenting on contributions published recently in the JRSM. They should not exceed 300 words and should be typed double spaced

\section{Diagnosis of perforated enterocystoplasty}

The data presented by Dr Fontaine and his colleagues (August $2003 J R S M^{1}$ ) suggest that perforation of an enterocystoplasty is often associated with an overfilled bladder which, in turn, can be produced by poor compliance with a strict intermittent catheterization regimen. It would be interesting to know how many of the total patients involved had diminished pain sensibility in their bladders, and what proportion of these were in the perforation group. Even the most fastidious patients may let their catheterization routine slip and this is a particular temptation when delay in performing catheterization does not result in pain.

It could be that such patients need an efficient 'pain substitute'. ${ }^{2}$ Perhaps urological surgeons should reconsider the plastic surgeon's technique (when repairing pressure ulcers) of using normally innervated autografts? Such a graft, using perhaps expanded skin, might one day be incorporated into reconstructed bladders that would otherwise have absent or diminished pain sensibility. I appreciate that this would not be an easy operation, and skin might not be the best tissue to use.

\section{Peter Lowthian}

134 Villiers Road, Oxhey,

Watford WD19 4FJ, UK

\section{REFERENCES}

1 Fontaine E, Leaver R, Woodhouse CRJ. Diagnosis of perforated enterocystoplasty. J R Soc Med 2003;96:393-4

2 Brand PW, Ebner JD. A pain substitute-pressure assessment in the insensitive limb. Am J Occup Ther 1969;23:379-486

\section{Living wills}

The care of the dying is an aspect of medicine that tests a doctor's skill, understanding and compassion more than any. There would be no demand for legalized euthanasia if the medical profession treated the dying as they should. Equally, there would be no call for 'living wills'. Acceptance by doctors of such documents as legally binding would be a form of defensive medicine, very much to the detriment of patient care. There is no place for the lawyer at the bedside of the dying.

The new Voluntary Euthanasia Society living will, described by Dr Michael Irwin (August 2003, JRSM,$^{1}$ ) provides two alternative choices-attempting to prolong life for as long as possible, or exclusion of life-sustaining treatment. Neither is compatible with acceptance of life as a precious gift and of death as a normal predictable event. Neither tends to a peaceful death. On the contrary, maybe I am cynical but I cannot help seeing in this a further attempt to increase people's fear of natural death and make them prefer euthanasia. Thus will they whose aim is to get rid of those whose life they consider to be worthless be able to say: 'It was their right and their wish to be helped to die'. Who benefits from a person writing a living will? Nobody does.

\section{B Howitt Wilson}

3 Brackendene Close, Horsell, Woking, Surrey GU21 4ED, UK

\section{REFERENCE}

1 Irwin M. A new kind of living will. J R Soc Med 2003;96:411

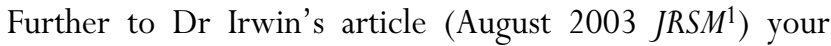
readers may wish to consider including in their Living Will three clauses that are relatively unusual.

Clause A: 'My family should stay out of this'. This Living Will indicates my wishes and directions and overrides, and does not in any way depend on, the contrary wishes, directions, opinions and preferences of any member of my family or any other person. It is directed to and is intended to bind, and I require that it be honoured by my family, my general practitioner, my attending consultant physician or my attending consultant surgeon, my lawyer, and by any individual who may become legally responsible for my health, welfare or affairs.

Clause B (compulsory supervision): Purported performance of this Living Will shall be invalid unless performance is actually and demonstrably directed and supervised by my attending consultant physician (if I am being treated for a medical condition) or my attending consultant surgeon (if I am being treated for a surgical condition).

Clause C (team of healthcare proxies): I appoint [names] or such of them as are available to make (whether in person or by telephone or other direct communication) binding healthcare decisions jointly on my behalf ("My Healthcare Proxies"). I require that the decisions of My Healthcare Proxies be made jointly, not severally or jointly-and-severally. No one or more of them shall have any power to make such decisions without all of the rest of them unless the missing one(s) cannot practicably be found after all diligent inquiry over a period of no less than seven working days. The responsibility for finding My Healthcare Proxies lies with my healthcare providers 
personally and individually. Their addresses and phone numbers are: [state].

\section{Richard Astor}

39A Wellbeck Street, London W1G 8DH, UK

E-mail: richardastor@astorlaw.com

\section{REFERENCE}

1 Irwin M. A new kind of living will. J R Soc Med 2003;96:411

\section{Patients' memory for medical information}

Dr Kessel (May $2003 J R S M^{1}$ ) discusses the very poor recall of medical information by patients after a consultation. In our ophthalmology department, the majority of patients are elderly. They are provided with both verbal and written information regarding their proposed cataract surgery on at least two occasions before surgery - at the time of listing for surgery and at preoperative assessment. Consent is obtained at the time of listing. At the time of admission they usually know the name of the proposed surgery and its beneficial effects but they seldom recollect information about its limitations or complications. In these circumstances, one begins to wonder whether the consent obtained is truly informed and whether it remains valid. With written information, poor vision perhaps contributes to poor recall.

\section{Jai Shankar}

Department of Ophthalmology, Wrexham Maelor Hospital, Wrexham LL13 7WX, UK

\section{REFERENCE}

1 Kessel RPC. Patients' memory for medical information. J R Soc Med 2003;96:219-22

\section{Medically unexplained symptoms}

Dr Page and Professor Wessely (May 2003 JRSM ${ }^{1}$ ) provide a useful analysis of actions by doctors that can exacerbate or sustain medically unexplained symptoms (MUS). However, one must never forget that one possible cause of 'medically unexplained' symptoms is the doctor's ignorance. Having spent many years dealing with patients with spinal pain (both neck and back) - and the non-operative treatment of it-I frequently see patients whose symptoms are 'unexplained' because others have not recognized that pain can be referred to distant parts (without signs of root pressure) other than the limbs (e.g. occipital headaches and facial pain from the upper cervical region, anterior chest pain from the upper thoracic region, upper abdominal pain from the mid-lower thoracic region and lower abdominal or groin pain from the upper lumbar or sacro-iliac regions). Furthermore, 'orthodox' medical teaching of clinical examination of the spine (and indeed the musculoskeletal system in general) is often poor or perfunctory, taking no account of the 'rhythm' or pattern of movement or localized restriction of movement and tenderness along with increased muscle tone (guarding).

Too many doctors, and now lawyers, want to rely on a 'negative scan' (either CT or MRI) to negate a physical diagnosis; or rely on a positive finding (which is common in asymptomatic people) to explain symptoms that are incompatible with the scan findings. They fail to realize that it is a question of 'altered physiology', rather than 'visible pathology', that is the cause of the symptoms; and that there is no need for such scans or X-rays in the majority of cases. (Might not the same be true for 'functional' problems in the gastrointestinal tract and cardiovascular system?)

In musculoskeletal medicine it is generally accepted that one should consider the 'bio-psychosocial' model, particularly in chronic spinal pain. In recent times the emphasis has been on the psychosocial aspects; but in most cases a genuine 'bio' element exists, if only people would look for it properly by good old-fashioned clinical examination.

\section{C T Morrison}

15 Prospect Hill, Swindon SN1 3JU, UK

\section{REFERENCE}

1 Page LA, Wessely S. Medically unexplained symptoms: exacerbating factors in the doctor-patient encounter. JRSM 2003;96:223-7 